\title{
Application of Electron Microscopy to Problems in the Growth of Laser Crystals
}

\author{
D. E. Eakins,* M. G. Norton,* D.F. Bahr,* J. Q. Dumm** \\ * Mechanical and Materials Engineering, Washington State University, Pullman WA 99164 \\ ** II-VI, Inc., 375 Saxonburg Blvd., Saxonburg PA 16056
}

Cracking of Czochralski grown Nd-doped YAG single crystals during growth becomes a major problem as the dopant concentration is increased. Dopant concentrations are typically up to a maximum of 1.5 substitutional $\% \mathrm{Nd}$. It is desired by commercial suppliers to grow large diameter crystals with even higher dopant concentrations in order to increase both laser efficiency and product yield. A knowledge of defects in commercially grown Nd:YAG is needed to understand some of the difficulties encountered during crystal growth.

Figure 1a shows a Nd:YAG boule containing $1.02 \% \mathrm{Nd}$ that had fractured during growth. The fracture originated at the crystal core and extended towards the crystal/melt interface. Examination of the fracture surfaces by scanning electron microscopy was undertaken to determine if any observable defects were present at the crack origin. Fractured boules were immersed in liquid nitrogen then placed in hot water to allow the fracture surfaces to extend to the free surface. River marks in the fracture surface, shown in Figure 1b, were used to trace the crack path back to its origin. These are indicative of screw dislocations threading through the plane of fracture [1]. In several cases, voids were observed at the crack origin, possibly leading to failure due to their role as stress concentrators. In others, voids were observed either removed from the crack origin or absent from the fracture surface altogether, suggesting failure was initiated by some other mechanism.

Electron transparent samples of $\mathrm{Nd}: \mathrm{YAG}$ were prepared using conventional mechanical methods with perforation being achieved by a chemical etch consisting of $1: 1 \mathrm{H}_{2} \mathrm{SO}_{4}$ and $\mathrm{H}_{3} \mathrm{PO}_{4}$ at $180^{\circ} \mathrm{C}$. Samples were prepared from boules of different dopant concentrations and at different regions within each boule. Transmission electron microscope examination of samples removed from the core region of a $1.02 \% \mathrm{Nd}$-doped sample contained particles ranging from $0.5-4 \mu \mathrm{m}$ in diameter [2]. Electron diffraction patterns obtained from the particles and surrounding matrix material indicated no structural or phase differences. (Compositional data could not be obtained using energy dispersive spectroscopy because of fluorescence of the samples during electron beam irradiation.) Moiré fringes were observed in high-resolution images of the particles as shown in Figure 1c. These arise as a result of interference and indicate either a difference in lattice parameter and/or orientation of the particles with respect to the matrix. The moiré fringe spacing was measured from the images to be $2.44 \mathrm{~nm}$ (Figure 1d). Analysis of the fringe spacing and direction allow determination of the interfering lattice planes. It was determined that the two planes were of the $\{211\}$ type, with spacings of $0.49049 \mathrm{~nm}$ and $0.49037 \mathrm{~nm}$ for the particle and matrix, respectively, and that the misorientation of the two sets of planes was $0.201^{\circ}$ (too small to be seen in SAD patterns). The change in lattice parameter with dopant concentration was determined using the precise lattice parameter method for X-ray diffraction [3]. Using these data and the results from the moiré fringe analysis it was shown that the dopant concentration in the particles was $2.768 \%$. The stress associated with these regions of strain was calculated to be $80 \mathrm{MPa}$. The actual stress fields will be complicated by varying particle sizes and distributions in the boule [4]. 
References

1. J. J. Gilman, Trans. Met. Soc. AIME 212 (1958) 310

2. D. E. Eakins et al., Proceedings of SPIE, 4970 (2003)

3. C. Suryanarayana and M.G. Norton X-Ray Diffraction: A Practical Approach, Plenum, New York (1998) p. 153

4. Financial support provided by the Department of Defense ASD/C3I (N66001-00-C-6008) through a subcontract from VLOC Inc. Part of this work was performed at the EMSL, a national user facility located at Pacific Northwest National Laboratory, operated for DOE by Battelle.

a
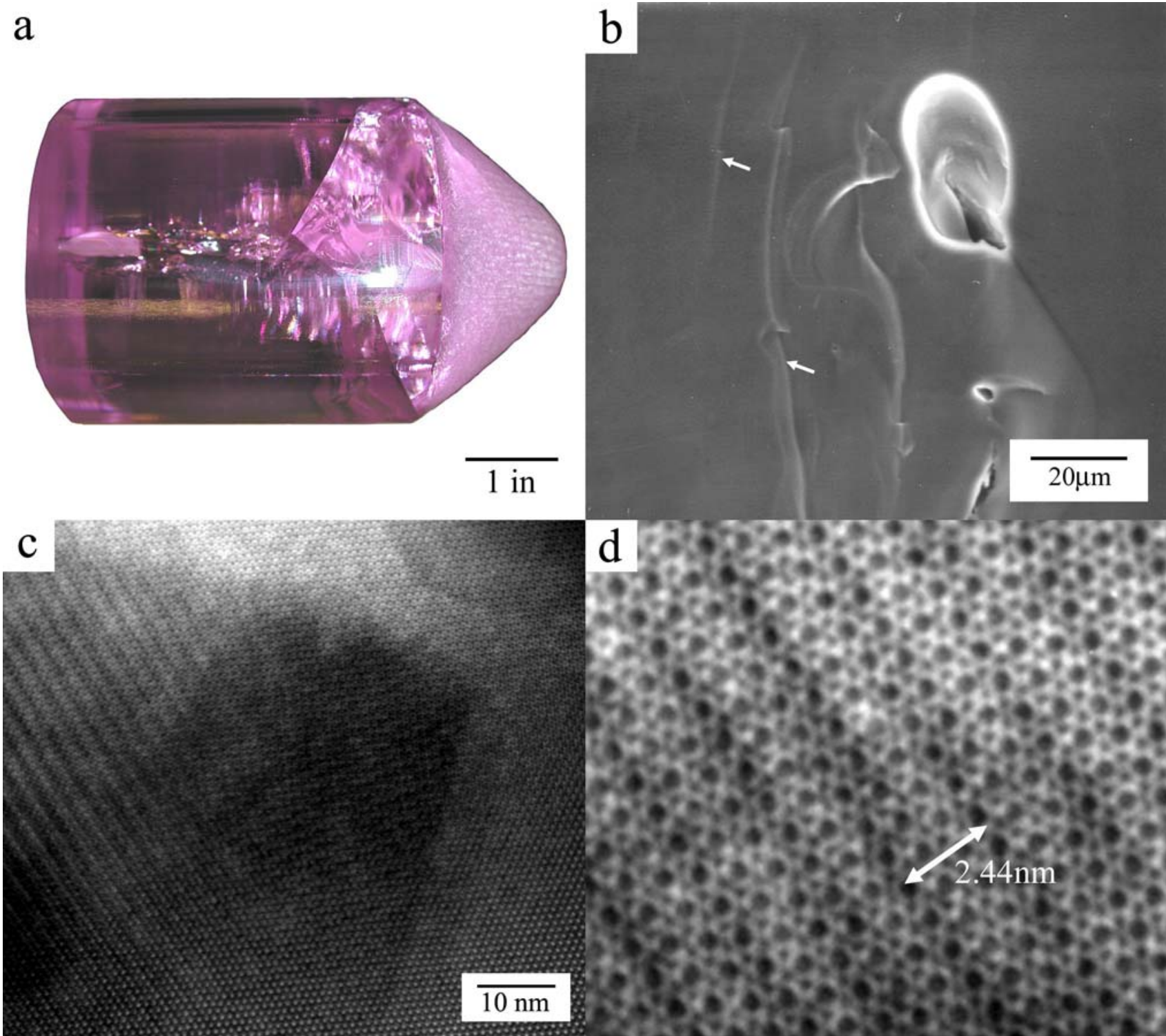

Figure 1 (a) Image of a fractured Nd:YAG boule. (b) SEM image of the YAG fracture surface showing rivermarks (indicated by arrows) traveling past a void. (c) High-Resolution image at the [111] zone axis of a particle exhibiting moiré fringes. (d) Magnified image of particle region showing fringe spacing. 